\title{
Global opportunities for mariculture development to promote human nutrition
}

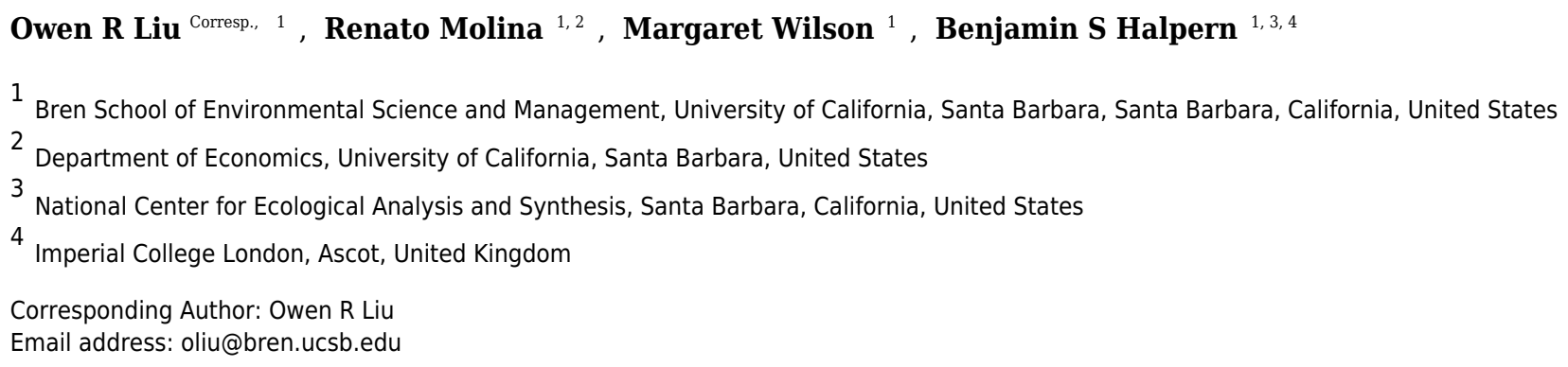

An estimated two billion people worldwide currently suffer from micronutrient malnutrition, and almost one billion are calorie deficient. Providing adequate nutrition is a growing global challenge. Seafood is one of the most important sources of both protein and micronutrients for many, yet production from wild capture fisheries has stagnated. In contrast, aquaculture is the world's fastest-growing food production sector and now supplies over half of all seafood consumed globally. Mariculture, or the farming of brackish and marine species, accounts for roughly one-third of all aquaculture production and has received increasing attention as a potential supplement for wild-caught marine fisheries. By analyzing global patterns in seafood reliance, malnutrition levels, and economic opportunity, this study identifies where mariculture has the greatest potential to improve human nutrition. We calculate a mariculture opportunity index for 117 coastal nations by drawing on a diverse set of seafood production, trade, consumption, and nutrition data. Seventeen primary variables are combined into country-level scores for reliance on seafood, opportunity for nutritional improvement, and opportunity for economic development of mariculture. The final mariculture opportunity score identifies countries with high seafood reliance combined with high nutritional and economic opportunity scores. We find that island nations in Southeast Asia and the Caribbean are consistently identified as countries with high mariculture opportunity. In other regions, nutritional and economic opportunity scores are not significantly correlated, and we discuss the implications of this finding for crafting appropriate development policy. Finally, we identify key challenges to ameliorating malnutrition through mariculture development, including insufficient policy infrastructure, government instability, and ensuring local consumption of farmed fish. Our analysis is an important step towards prioritizing nations where the economic and nutritional benefits of expanding mariculture may be jointly captured. 


\section{Global opportunities for mariculture development to promote} 2 human nutrition

3

4

5

6 7

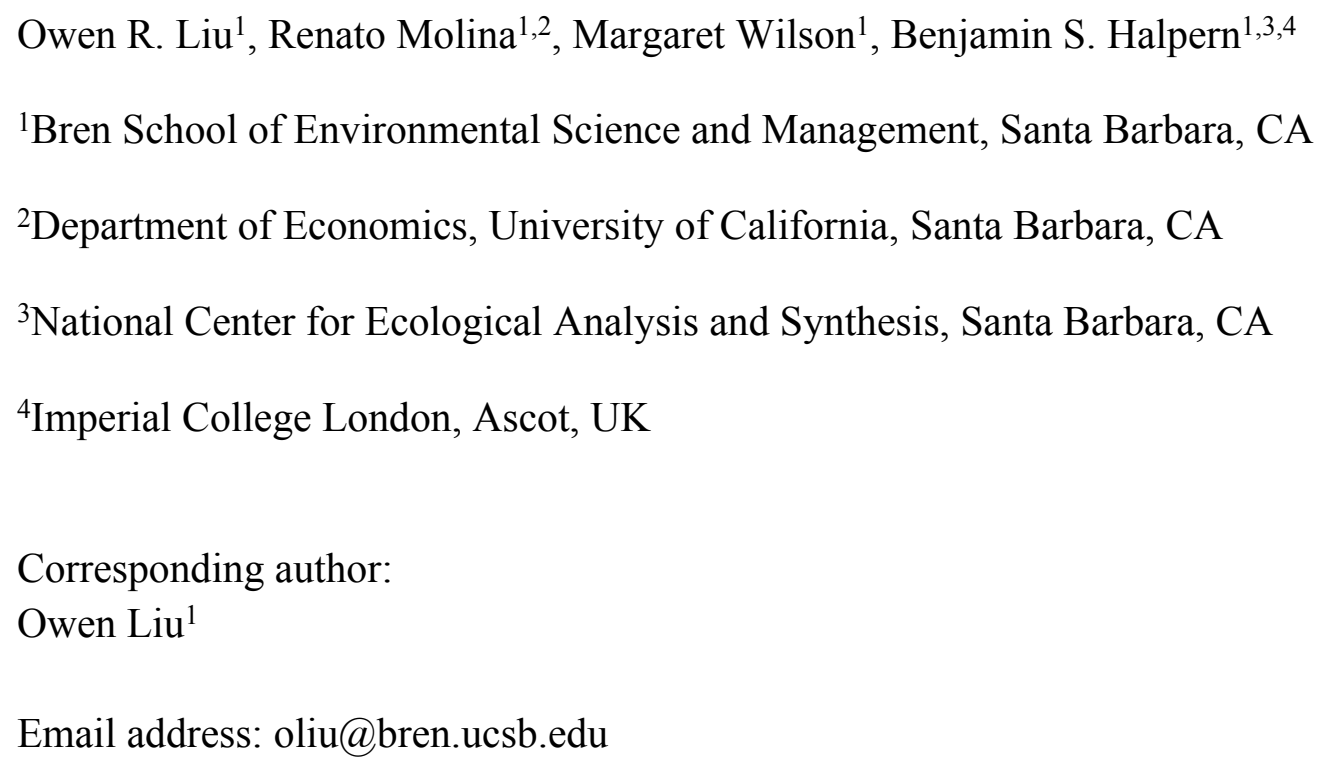

Email address: oliu@bren.ucsb.edu 
20

21

22

23

24

25

26

27

28

29

30

31

32

33

34

35

36

37

38

39

40

41

42

43

44

45

46

47

48

49

\title{
Global opportunities for mariculture development to promote human nutrition
}

\author{
AUTHORS: Owen R. Liu ${ }^{1}$, Renato Molina ${ }^{1,2}$, Margaret Wilson ${ }^{1}$, Benjamin S. Halpern ${ }^{1,3,4}$ \\ ${ }^{1}$ Bren School of Environmental Science and Management, Santa Barbara, CA \\ ${ }^{2}$ Department of Economics, University of California, Santa Barbara, CA \\ ${ }^{3}$ National Center for Ecological Analysis and Synthesis, Santa Barbara, CA \\ ${ }^{4}$ Imperial College London, Ascot, UK
}

\section{ABSTRACT}

An estimated two billion people worldwide currently suffer from micronutrient malnutrition, and almost one billion are calorie deficient. Providing adequate nutrition is a growing global challenge. Seafood is one of the most important sources of both protein and micronutrients for many, yet production from wild capture fisheries has stagnated. In contrast, aquaculture is the world's fastest-growing food production sector and now supplies over half of all seafood consumed globally. Mariculture, or the farming of brackish and marine species, accounts for roughly one-third of all aquaculture production and has received increasing attention as a potential supplement for wild-caught marine fisheries. By analyzing global patterns in seafood reliance, malnutrition levels, and economic opportunity, this study identifies where mariculture has the greatest potential to improve human nutrition. We calculate a mariculture opportunity index for 117 coastal nations by drawing on a diverse set of seafood production, trade, consumption, and nutrition data. Seventeen primary variables are combined into country-level scores for reliance on seafood, opportunity for nutritional improvement, and opportunity for economic development of mariculture. The final mariculture opportunity score identifies countries with high seafood reliance combined with high nutritional and economic opportunity scores. We find that island nations in Southeast Asia and the Caribbean are consistently identified as countries with high mariculture opportunity. In other regions, nutritional and economic opportunity scores are not significantly correlated, and we discuss the implications of this finding for crafting appropriate development policy. Finally, we identify key challenges to ameliorating malnutrition through mariculture development, including insufficient policy infrastructure, government instability, and ensuring local consumption of farmed fish. Our 
50 analysis is an important step towards prioritizing nations where the economic and nutritional

51 benefits of expanding mariculture may be jointly captured.

\section{INTRODUCTION}

54

With large uncertainty surrounding the future of wild caught fisheries, the potential role of farmed fish has gained increasing attention in global nutrition conversations (Beveridge et al. 2013; Béné et al. 2015; Golden et al. 2016; Little et al. 2016). An estimated one billion people are calorie deficient, and two billion suffer from micronutrient malnutrition (IFPRI 2016). Zinc deficiency affects $17 \%$ of the global population (Golden et al. 2016) and is responsible for an estimated 800,000 annual child mortalities (FAO 2016). Nearly one-third of the world's population is iron deficient (FAO 2016) and one-fifth of maternal deaths are linked to anemia during pregnancy (Micronutrient Initiative 2009). Vitamin A deficiency is the leading cause of preventable blindness and affects an estimated 250-500 million children, half of whom will die within a year of vision loss (Bailey et al. 2015).

Seafood is a critical source of all of these nutrients. Fish currently provides $17 \%$ of the world's animal protein, and exceeds 50\% in the diets of many least-developed countries (FAO 2016). acids found in fish and their contribution to brain development, metabolic function, and the prevention of cardiovascular disease (Larsen et al. 2011). But seafood in general also provides essential micronutrients that promote healthy growth and development, particularly in children and pregnant women (Kawarazuka and Béné 2011; Béné et al. 2015; FAO 2016). Nevertheless, declines in global wild fish stocks paired with a predicted human population of nearly 10 billion by 2050 may leave even greater numbers at risk of nutrient deficiency (UNDP 2015; Blasiak et al. 2017). Golden et al. (2016) estimate that an additional $11 \%$ of the population is vulnerable to zinc, iron, and vitamin A deficiencies as fish stocks decline in coming decades, and nearly $20 \%$ 78

Due in part to the nutritional importance of fish and significant technological advances to 80 produce seafood (Kumar and Engle 2016), its consumption has more than doubled from $9.9 \mathrm{~kg}$ 
81 per capita in the $1960 \mathrm{~s}$ to a current average of $20.2 \mathrm{~kg}$ (FAO 2016). Global fish consumption is

82 predicted to increase more than $20 \%$ by 2025 , as both human population and economic

83 development rise in coming decades (FAO 2016). Driven by this increasing demand, aquaculture

84 has been the fastest growing food production sector for four decades and now exceeds wild

85 fisheries production (Tveteras et al. 2012; Troell et al. 2014). About one-third of this total

86 production comes from the farming of marine species, also known as mariculture (Ottinger et al.

87 2016). While issues around freshwater scarcity (Verdegem and Bosma 2009) and pollution (Cao

88 et al. 2007; Edwards 2015) may slow the growth of freshwater aquaculture in coming years,

89 mariculture has been identified as an area of high growth opportunity (Holmer 2010; Kapetsky et

90 al. 2013; Gentry et al. 2017).

91

92 Increased mariculture production could help ameliorate global malnutrition, but its current

93 development typically excludes lower-income countries or is marketed towards trade with

94 wealthier countries and consumers (Watson et al. 2015; Asche et al. 2015a; Golden et al. 2016,

95 2017). Global mariculture production currently focuses predominantly on high-value species like

96 salmon, shrimp and tuna, which largely go to global markets (Bostock et al. 2010). It remains

97 unknown whether mariculture can meaningfully contribute to global nutrition, in part because no

98 previous analysis has identified countries where economic and nutritional development

99 opportunities are expected to overlap. Before developing any strategies to link these objectives,

100 however, it is critical to first identify key overlaps between nutritional needs and economic

101 opportunity for further mariculture development.

102

103 Here we provide global analyses to identify countries where joint economic and nutritional

104 mariculture development may be most synergistic. Our motivating question is, where do

105 nutritional needs — needs that can be effectively alleviated by seafood consumption — overlap

106 with economic development opportunities for mariculture? By using global datasets and

107 developing a comparative scoring system, we identify high-opportunity countries via an analysis

108 of country-level malnutrition, seafood reliance, and economic opportunity. We dissect emergent

109 patterns in the global analysis and discuss their potential drivers. Finally, we identify common

110 development obstacles that may be applicable to future global mariculture ventures. 


\section{METHODS}

114

\section{Defining mariculture opportunity}

117 For a country to tackle the nutritional deficiencies of its population through mariculture

118 development, it should have three main characteristics, expressed herein through three scores 119 that we compile for each nation in our analysis. First, the country should have a demonstrated 120 need for the macro- and micronutrients that seafood can provide. As described above, seafood 121 can be an efficient and important source of not just calories, but also protein, healthy fatty acids, 122 zinc, vitamin A, and iron (Kawarazuka and Béné 2011; Béné et al. 2015). On the other hand, 123 countries that are well-nourished will not necessarily benefit (nutritionally) from adding more 124 fish to the diet. We refer to a country's relative deficiencies in these key nutrients as the 125 country's nutritional opportunity.

126

127 There should also be good evidence within the country of a cultural predisposition to seafood 128 consumption. Clearly, increases in mariculture production will be most directly important for 129 alleviating nutritional deficiencies if seafood accounts for a large proportion of a country's diet. 130 For this reason, we also include seafood reliance - calculated as the relative contribution of 131 seafood to total diet - as a core enabling factor for mariculture opportunity.

Finally, a country's mariculture production should be economically viable in order to sustainably provide a nutritional solution. Many combined mariculture/development projects fail to be sustainable because of a lack of scalability or long-term economic feasibility (Béné et al. 2016; Little et al. 2016). Hence, our third score for each country is a measure of this economic opportunity, constructed from each country's current aquaculture production and seafood trade data, as well as proxies for the value of the seafood production sector and latent economic development potential. 
143 We compiled raw data for economic opportunity, nutritional opportunity, and seafood reliance

144 from two publicly available databases (Figure 1). We endeavored to limit our metrics to those

145 that are directly relevant to the economic development of mariculture and the alleviation of

146 nutritional deficiencies through seafood production. The resulting set of raw data includes five

147 economic opportunity metrics, six nutritional opportunity metrics, and six seafood reliance

148 metrics by country. To facilitate global comparisons, these 17 raw metrics were normalized and

149 then combined into the three opportunity metrics and a final mariculture opportunity metric

150 (Figure 1).

151

152 Nutritional opportunity and seafood reliance scores were calculated using raw metrics from the

153 Harvard GENuS database (Smith et al. 2016, https://dataverse.harvard.edu/dataverse/GENuS).

154 GENuS models comprehensive country-specific diet and nutrient supply information by

155 extrapolating from the Food and Agriculture Organization of the United Nations' (FAO) food

156 balance sheets, household surveys, and production data. GENuS estimates per capita nutrient

157 consumption across hundreds of food categories. For the purposes of this study, we utilized data

158 on the average daily per capita intake by country of five essential nutrients that can be obtained

159 from seafood: protein, vitamin A, zinc, iron, and polyunsaturated fatty acids (PUFAs).

160 Separately, we also collected each country's average Dietary Energy Supply Adequacy, an FAO

161 measure of the basic adequacy of total caloric intake relative to a sufficient diet (Eq. 1, 2). These

162 data - energy adequacy plus the daily per capita intake of five nutrients - comprise our six

163 nutritional opportunity metrics. Together, these measures provide a synthesis of the average

164 nutritional status of each country, specific to those nutrients that mariculture products can

165 provide.

166

167 Our raw seafood reliance data were also drawn from the GENuS database. Because GENuS

168 provides per capita nutrient intakes by food-group, we were able to sum per capita intakes from

169 all FAO marine harvest categories (pelagic fish, demersal fish, other marine fish, crustaceans,

170 and mollusks) to calculate total nutrient and calorie intakes obtained from seafood. We divided

171 these seafood-specific intake values by total per capita intake values to calculate the percent of

172 each nutrient obtained from seafood products. Six of these percentage values - for calories,

173 protein, vitamin A, zinc, iron, and PUFAs - comprise our six seafood reliance metrics. Having 
174 both average nutritional status (nutritional opportunity score) and seafood reliance allows our

175 scoring system to identify countries where increased mariculture production may have the

176 greatest chance to directly address nutritional deficiencies, and where vulnerability to potential

177 declines in wild-caught fisheries is highest.

178

179 The third dimension of mariculture opportunity is economic opportunity. Economic metrics were 180 drawn from FishStatJ (http://www.fao.org/fishery/statistics/software/fishstatj/en), a freely 181 available software used to access data from the Fisheries and Aquaculture division of FAO.

182 FishStatJ provides panel data on fisheries and aquaculture production and trade by country, 183 species, and commodity type. Selecting only the most recent year for which all metrics are 184 available (2011), and excluding all commodity categories not for direct human consumption 185 (e.g., fish meal or fish oil), these data were analyzed to produce the five economic opportunity 186 metrics for each country: 1) production ratio, 2) trade balance in terms of quantity, 3) trade 187 balance in terms of value, 4) GDP per capita, and 5) willingness to pay for seafood.

189 We define a country's production ratio as its total aquaculture production divided by its total 190 marine fisheries production in metric tons. Both production metrics were drawn directly from 191 FAO reported data. This measure serves as a proxy for relative importance of two sectors that 192 share infrastructure and markets. The logic is that countries with active fishing sectors should 193 have both capital and management institutions that could also be functional to production and 194 regulation of mariculture. The balance of fisheries and aquaculture production determines the 195 opportunity for mariculture to utilize that shared infrastructure. The more skewed the production ratio is toward fisheries, the more potential there is to take advantage of these overlaps through the further development of a mariculture sector. While an indirect proxy for infrastructure, production ratio was chosen because of its generality across multiple types of potential mariculture production and its consistency across countries.

201 Two of our economic opportunity metrics measure trade balance in quantity and value. In our 202 study, trade balance describes each country's total volume or value of exports of seafood 203 products (not just mariculture) divided by its imports. Trade balance measured in this way is a 204 proxy for how a country balances supply and demand in the global seafood market. Trade 
205 imbalances reveal how countries compensate for their domestic seafood demand: a trade

206 imbalance in which imports outweigh exports implies an opportunity to satisfy excess demand

207 with augmented domestic mariculture production. Because seafood products vary so widely in

208 their value relative to their volume, this trade balance signal could manifest in either metric,

209 hence our inclusion of both quantity and value metrics.

210

211 The metric for GDP per capita is included as a proxy for latent economic opportunity. Countries

212 with low per capita GDP have a need for economic development that may be partially pursued

213 through mariculture. In this way, lower GDP per capita corresponds to higher economic

214 opportunity scores in our analysis.

215

216 Willingness to pay for seafood, our final economic opportunity metric, is defined as a country's

217 total value of seafood imports divided by its GDP. This measure serves as a proxy for seafood

218 value in each country. Although an imperfect metric, as it combines high-volume, low-value

219 seafood with high-value niche products, willingness to pay still reflects overall expenditure on

220 foreign seafood production. In our analysis, a higher willingness to pay corresponds to a greater

221 opportunity to capture that willingness to pay with mariculture products produced domestically.

222 In the calculation of opportunity scores in the next section, we use the reciprocal value of

223 willingness to pay so that its ordering aligns with the other economic metrics (a lower value of

224 the metric corresponds to a higher economic opportunity).

225

226 Together, these economic measures provide the essential information to describe a given 227 country's current mariculture production status relative to other nations. Furthermore, by 228 utilizing FAO data, this set of economic metrics provides the ability to contrast countries while 229 reducing potential sources of inconsistency and bias that might arise from using disparate 230 sources, while at the same time being readily amenable to update as new data become available. 231 Each individual metric provides one perspective on the enabling conditions for economic 232 development of mariculture. Based on our economic opportunity metrics, a country with a high 233 economic opportunity is one with existing seafood industry infrastructure, a seafood trade 234 balance that could benefit from increased domestic production, a demonstrated value of seafood 235 in the country, and a relatively low per capita GDP. 


\section{Calculation of mariculture opportunity scores}

238

239 The 17 metrics were combined into three opportunity scores and one final mariculture 240 opportunity score (Fig. 1). All raw metrics were normalized to a zero to one scale to allow 241 comparison across categories of metrics. Each metric was scaled by dividing by its 80 th 242 percentile value across countries (Eq. 1).

$$
X_{i}=\frac{X_{\text {raw }, i}}{P_{80}\left[X_{\text {raw }}\right]}
$$

245 This scaling was chosen to reduce the influence of large single-metric outliers on the ability to 246 distinguish between nations. The choice had little effect on the final ranks of nations compared to 247 dividing by the $90^{\text {th }}$ percentile or simply the maximum value for each metric (see Appendix 248 Table A2; final country ranks between three alternate scaling choices were significantly 249 concordant; Kendall's $W=0.925, p<0.05)$.

250

251 The three scores - nutritional opportunity, seafood reliance, and economic opportunity — for each 252 nation were determined by calculating a mean across the set of normalized metrics associated with that score (Eqs. 2-4, Fig. 1). No weighting was done in the calculation of aggregated scores because our emphasis is on countries' relative positions. There was no definitive rationale for weighting any metric more heavily than any other, and doing so might unnecessarily complicate the interpretation of our scoring system and results. While we did not choose to use weighted scores, our methodology remains flexible to that extension.

259 Equations 2-5 describe score calculation. Country i's economic opportunity score (Eq. 2) was 260 defined as the mean of its normalized metrics for production ratio, trade balance in value, trade balance in quantity, willingness to pay, and GDP per capita. The country's nutritional opportunity score is the mean of its normalized intakes of protein, vitamin A, zinc, iron, and PUFAs, as well as its normalized FAO energy adequacy. Because the raw dietary nutritional supplies do not scale to zero - no country's diet consists of zero calories - the set of nutritional 
266 by the range across all nutritional opportunity scores. Finally, a country's seafood reliance score 267 is the mean of its normalized metrics for protein, vitamin A, zinc, iron, fatty acids, and calories 268 derived from seafood. Each of the three metrics was ordered such that a higher score (closer to 1) 269 corresponds to higher opportunity in that dimension, as described in the previous section.

270

271

272

273

274

275

276

277

278

279

280

281

282

283

284

285

286

287

288

289

290

291

292

293

294

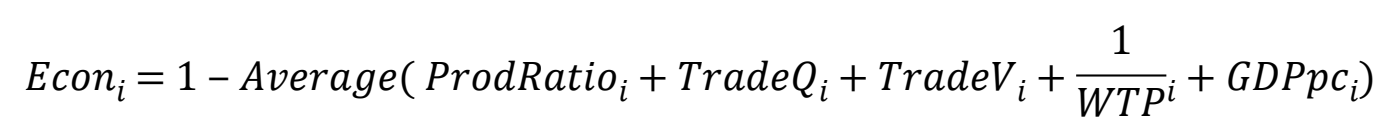

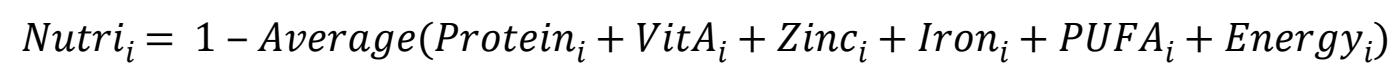

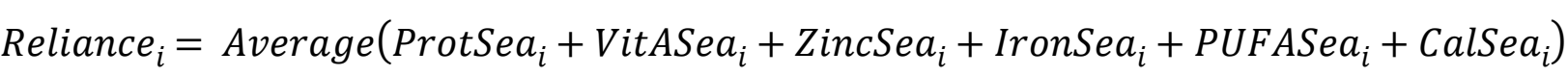

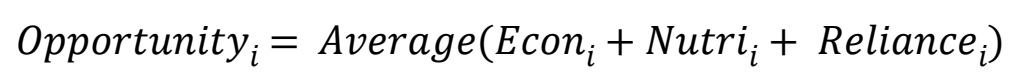

A final mariculture opportunity score for each country was calculated by averaging its economic opportunity, nutrition opportunity, and seafood reliance scores (Eq. 5).

Non-coastal nations $(\mathrm{N}=49)$ were excluded from the analysis before score calculation, because we were focused on the potential for local mariculture to address in-country nutritional needs. We likewise removed countries missing entire categories of data (e.g., missing all nutritional data). To avoid overly biasing our sample against data-limited countries, we retained countries with missing aquaculture production $(\mathrm{N}=23)$, dietary energy supply adequacy $(\mathrm{N}=10)$, and GDP $(\mathrm{N}=4)$ values and simply omitted those individual variables from the countries' score calculations. Sensitivity analyses on nations with complete data revealed that the effect on countries' opportunity scores of single missing metrics was minimal, so gap-filling procedures (and their associated uncertainty) were not deemed necessary (Table A3). The final sample 
295 consists of 117 coastal nations. All raw and normalized metrics and scores for each country are 296 available in the supplementary data.

297

298

\section{Limitations of the analysis}

299

300 The metrics described in the section above have two main advantages: i) the data required is 301 readily available through standard and credible data sources, and ii) the calculation of each 302 metric is straightforward and amenable to further refinement. However, the metrics also have 303 two important limitations that warrant mention, and both are fully explored in the Appendix. 304

First, our metrics are constructed with cross-sectional data. This feature impedes our ability to 306 explicitly examine past trends or predict future trajectories. But any cross-sectional approach suffers from the same limitations, and we are forced to rely on the assumption that the data reflects the actual realities of every country for that specific point in time.

Particular to our case, the limiting factor is that data for nutritional intakes (GeNUS) are only available for 2011. If a panel dataset on nutritional intake were to be available, it may prove beneficial to work with trends by country, rather than single observations for specific years. Unfortunately, to the best of our knowledge, these data are unavailable. We therefore use crosssectional (2011) data in our main analysis, but in the Appendix we provide an extension to test the sensitivity of our results to the economic data, which are available in time series.

317 Secondly, it is possible that our seafood reliance score reflects preferences rather than pure 318 reliance. For example, rich countries showing high seafood reliance could be merely a result of 319 the average consumer's preferences and may have little to do with the overall seafood availability or its price. If the index reflects preferences rather than pure reliance, the interpretation of the seafood reliance may be altered. This is an important issue if we are trying to establish the potential of mariculture in solving nutritional needs, but at the same time it is intrinsic to how these aggregate measures are collected across nations. We address this limitation in the Appendix by extending our main analysis and sorting countries in the sample based on their income level as reported by the World Bank (Fig. A3), as well as their geographical 
326

327 328

329

330

331

332

333

334

335

336

337

338

339

340

341

342

343

344

345

346

347

348

349

350

351

352 353

354 355 356

characteristics (Continental or Island; Fig. A2). Breaking down our results in this manner allows us identify the relative influence of income and country type on seafood reliance.

\section{RESULTS}

Final mariculture opportunity scores are mapped in Figure 2. Countries with high mariculture opportunity scores (orange and red) are places where mariculture has the highest potential to ameliorate nutritional deficiencies because of the apparent alignment between nutritional and economic opportunities and a demonstrated reliance on seafood. These multi-dimensional opportunities are apparent for some nations, especially island nations in the Caribbean (Fig. 2B) and Southeast Asia. Other nations, notably in parts of Europe and Africa, also had high mariculture opportunity scores.

In addition to geographic patterns in final mariculture scores, several patterns emerged for each of the three separate opportunity scores (Fig. 3). First, countries' economic opportunity scores were generally clustered towards greater opportunity (mean score $0.59+/-0.2$, Fig. 3 and S1). This pattern indicates that comparatively few nations have developed mariculture industries, while the bulk of nations have potential to further develop mariculture. Indeed, the few top producers received low economic opportunity scores. China, Indonesia, the Philippines, and Norway, which are four of the five top current producers of mariculture (FAO 2016), all received scores less than 0.35 . Overall, mariculture production level was significantly negatively correlated with economic opportunity (Pearson's $r=-0.22, p<0.05$ ).

In contrast, relative to the economic scores, nutritional opportunity scores were generally lower, or right-skewed (mean nutritional opportunity score 0.42 +/- 0.23, Fig. 3). Europe and Asia were the regions with the overall lowest nutritional opportunities, while the Southeast Asia/Oceania and Latin America/Caribbean regions show the highest nutritional opportunity.

This combination of higher economic opportunity and lower nutritional opportunity creates the cluster of nations in the lower right quadrant of Figure 3. Fifty-three of 117 nations, or 45\%, have an economic opportunity score greater than 0.5 and a nutritional opportunity score less than 
357 0.5. Overall, economic and nutritional opportunity scores are positively correlated, but the 358 correlation is not significant (Pearson's $r=0.15, p=0.1$ ).

359

360 Nutritional opportunity and seafood reliance show significant positive correlation overall

361 (Pearson's $r=0.23, p=0.01$ ). Nine of the top 10 country scores (and 15 of the top 20) for

362 seafood reliance come from island nations. Countries with a higher reliance on seafood generally

363 have higher nutritional opportunity scores, although there were differences in the relationship

364 between reliance and nutrition between geographic regions. Southeast Asian countries (purple 365 dots in Fig. 3) have generally high nutritional opportunity and high seafood reliance, but scatter 366 along a spectrum of economic opportunity. In contrast, Latin American and Caribbean countries

367 (teal dots) display a high correlation between nutritional opportunity and seafood reliance: low 368 nutritional opportunity corresponds with low reliance, and vice versa. European countries display 369 a range of seafood reliance, but generally low nutritional opportunity.

370

371

\section{DISCUSSION}

372

373 The objectives of our analysis were to identify opportunities for nutritional improvement through 374 mariculture and to use multidimensional opportunity scores to inform future development efforts.

375 Our results identify countries with poor nutrition and high reliance on seafood. Of those

376

377

378

379

380

381

382

383

384

385

386

387 countries, our overall mariculture opportunity score prioritizes those with simultaneous large nutritional and economic opportunities for mariculture development. Other analyses have projected large increases in production in many of the nations - especially in Latin America, the Caribbean, and Southeast Asia — which we identified as having high nutritional opportunity (Kobayashi et al. 2015). Mariculture has the potential to benefit malnourished populations in these countries both directly through increasing seafood availability and indirectly through economic gains (Béné et al. 2016), and our analysis clearly identifies places where these opportunities exist. But despite this theoretical potential, limited evidence exists suggesting mariculture will address local nutritional needs in reality (Beveridge et al. 2013; Béné et al. 2016; Golden et al. 2016, 2017; Little et al. 2016). What barriers are preventing the potential nutritional benefits of mariculture from being realized, and how can we use this global analysis to guide nutritionally focused development strategies? 
388

389 The link between mariculture, or aquaculture in general, and the amelioration of malnutrition has

390

391

392

393

394

395

396

397

398

399

400

401

402

403

404

405

406

407

408

409

410

411

412

413

414

415

416

417

418

not proven inherent (Golden et al. 2017). Numerous countries have already developed fish

farming industries but still struggle with malnutrition. Our analysis corroborates this disconnect

by finding many nations with a low economic opportunity but high seafood reliance and

nutritional opportunity (upper left quadrant in Fig. 3). In these scenarios, aquaculture production

is not being translated into nutritional gains for at-risk populations. This widespread disconnect

between aquaculture production and local nutrition is largely a reflection of the industry's

historical development. Private investment opportunities - as opposed to nutritional necessity-

have been the primary drivers of aquaculture growth, especially in recent decades (Little et al.

2016). As industries have developed, improved productivity of larger farms and increased international trade have incentivized consolidation and export-oriented operations in many

producing countries (Asche et al. 2015b; Little et al. 2016).

This trend, however, does not necessarily mean that growth in mariculture production is or will be completely at the expense of the malnourished poor. Toufique and Belton (2014) found a convincing positive link between large-scale growth of pond aquaculture in Bangladesh and fish consumption by the extreme poor. Although the debate surrounding the strength of the link between mariculture development and nutrition improvement remains active, it is clear that the two are not necessarily mutually exclusive. Our analysis provides a guide to the countries where this link might be more effectively forged.

In this context, opportunity costs may pose a major barrier to nutritionally-focused mariculture development. Small island nations were overwhelmingly identified as high-opportunity countries in our analysis. Many of these nations, especially in the Caribbean, are on a development path focused on the promotion of tourism and importation of wealth from abroad (Ashe 2005; Scheyvens and Momsen 2008). Because of the tourism industry, coastal property is at a premium, turning coastal or near-shore mariculture activities into a non-competitive investment alternative. A shortage in affordable coastal real estate might incentivize development of offshore mariculture, though these systems will require significant amounts of external investment and technological capacity. To stay profitable, high cost systems will likely focus on 
419 high market value species intended for export and/or consumption by higher income individuals.

420 Several mariculture initiatives in the Caribbean have already been designed in this manner. In

421 Antigua, for example, a private mariculture initiative led by an American and European board of

422 directors plans to develop "high-tech" offshore pens to raise Kampachi (Seriola rivoliana), a

423 high value, sashimi grade fish for export (http://www.asacip.com/). While these projects

424 typically promise to provide local employment, the expected outcome for local nutrition remains

425 unclear (Béné et al. 2016). Hence, while our analysis may identify island nations as an important

426 focus for mariculture development and nutrition, capturing this overlapping opportunity will be a

427 policy challenge unique to each country or project.

428

429 In cases where mariculture products are made locally accessible, effectively addressing

430 malnutrition issues also requires significant education and marketing programs at local to

431 regional scales. Our opportunity criteria prioritized countries with high existing seafood reliance.

432 Existing culture around eating seafood in these countries may facilitate a transition to consuming

433 mariculture products, though local attitudes towards farmed fish may prove a significant barrier.

434 Case studies in Bangladesh reveal that farmed fish are typically harvested at a larger size and

435 consumed filleted, which may provide less nutritional value than the small indigenous fish that

436 are traditionally consumed whole (Kawarazuka and Béné 2011). Nutrition programs can play a

437 critical role in educating the public on product selection and preparation in order to maximize

438 nutritional effectiveness.

439

440 While our chosen scoring system prioritizes countries with high seafood reliance, there are

441 certainly opportunities to link mariculture production with local nutritional benefit when reliance

442 is low, and dietary contribution of seafood may increase with development (see Appendix).

443 These efforts, however, will require even greater investment in social planning and policymaking

444 to ensure these products are reaching nutritionally vulnerable populations. Alternatively, the

445 direct involvement of poor sectors in the mariculture industry could increase disposable income

446 and, consequently, access to nutritious food. This was demonstrated for aquaculture in Malawi

447 (Aiga et al. 2009), though these types of indirect benefits require further investigation (Béné et

448 al. 2016; Golden et al. 2017).

449 
450 A further challenge in mariculture development is to mitigate environmental harm to the extent

451 possible. A recent study establishes that there are vast areas suitable for development of

452 mariculture in almost every coastal nation (Gentry et al. 2017). Nonetheless, while suitable space

453 is likely not limited, intensive mariculture development comes with a host of potential

454 environmental problems, including pollution, habitat destruction, and disease risk to wild fish

455 populations (Klinger and Naylor 2012). Environmental harm from the development of

456 mariculture risks exacerbating some of the same human health factors it would seek to alleviate

457 (Cole et al. 2009). Best practices for mariculture development are rapidly being developed and

458 refined, and should be incorporated as an additional consideration in any nutrition-focused

459 mariculture development (Klinger and Naylor 2012).

460

461 Choosing appropriate mariculture species and practices will have significant implications not

462 only for environmental impacts but also for nutritional quality and accessibility. Seafood

463 products vary greatly in their specific nutrient content and therefore their potential contributions

464 to human nutritional needs (Glencross 2009, Hixon et al. 2014). A case study in Indonesia

465 demonstrates the potential impact of farming a particularly vitamin A-rich species on local

466 vitamin A deficiencies (Fiedler et al. 2016). Husbandry practices also have major impacts on

467 nutritional quality, as well as environmental impacts. Species-specific feed compositions that

468 provide digestible and appropriately-balanced nutrient compositions can improve fish growth

469 and nutritional value while also minimizing waste due to nutrient indigestibility or oversaturation

470 (National Research Council 2011, Hixon 2014). Because fish feed is the primary contributor to

471 aquaculture's negative environmental impacts, improved practices that minimize overfeeding are

472 critical in mitigating these deleterious effects (Boyd et al. 2007). Choosing local species can

473 reduce the risk of invasive escapes as well as the spread of disease (Diana et al. 2013).

474 Husbandry improvements such as reduced stock densities and appropriate water circulation can

475 also reduce disease outbreaks and lessen the need for antibiotics, improving product quality for

476 human consumers and alleviating environmental issues with antimicrobial resistance (Huer et al.

477 2008, Diana et al. 2013). As introduced earlier, the species produced in a given mariculture

478 initiative can also determine seafood accessibility, with the nutritional and economic benefits of

479 high-value species often failing to reach in-need populations (Golden et al. 2017).

480 
481 The ability of a country to develop nutritionally-sensitive mariculture production will be 482 extremely dependent on national policy and governance. Thilsted et al. (2016) advocate for 483 'nutrition-sensitive' fisheries and aquaculture policy that prioritizes context-specific nutritional 484 needs and preferences. Policy incentivizing production of locally consumed, affordable, and 485 nutritious products will be needed to prevent the dominant trajectory of export-oriented 486 mariculture. Education and accessibility programs will also need policy support. Unfortunately, 487 the overall mariculture opportunity score in our analysis is significantly correlated with the 488 World Governance Indicator for political stability from the World Bank 489 (http://info.worldbank.org/governance/wgi). This relationship means that countries needing 490 nutritionally sensitive mariculture the most are also those with potentially the least capacity for implementation. This issue, however, is also an opportunity. The substantial overlap between economic and nutritional scores in our analysis suggests that in many countries, well-designed mariculture development programs and policies should be able to tackle both poverty alleviation and nutrition improvement outcomes.

There was not a significant correlation between nutritional and economic opportunity scores in our analysis, meaning that countries with high nutritional opportunity may not necessarily be places that can (or should) address these nutritional needs through further mariculture development. Instead, nutritional improvement in countries with highly developed mariculture industries may face more of a distributional rather than a production challenge (Asche et al. 2015a; Watson et al. 2016). Indonesia, for example, has a high nutritional need (nutritional opportunity score 0.86), while being the world's second-largest aquaculture producer. Strategies to better link mariculture and local nutrition in countries like Indonesia should consider existing mariculture industries and take advantage of them to the extent possible. Potential approaches include the transition of existing infrastructure or sharing processing facilities. Policy and market-based incentives would be critical in incentivizing a partial shift from export-oriented products to more accessible, low value species that could be sold and consumed domestically. Our analysis identifies the countries where this shift may be beneficial.

509

510 Finally, it is important to note that our analysis does not capture all of the nuances associated 511 with the prospects and feasibility of mariculture development across the world. Institutional 
512 settings and market innovations may play a primary role when it comes to the actual

513 development of technology and aquaculture (Asche and Smith 2018). Further analysis and

514 understanding of local and national-level institutions will be critical for effective mariculture

515 development. Additionally, our global analysis, by necessity, is based upon data aggregated and

516 averaged at the country level and may miss within-country as well as over-time dynamics. For

517 example, just because a nation scores low on our nutritional opportunity scale does not

518 necessarily mean there are not vulnerable segments of the population. South Africa, for

519 example, receives a nutritional opportunity score of just 0.24 . But it is also a nation of extreme

520 inequality: The bottom $20 \%$ of the population receives less than $5 \%$ of national income, while

521 the top 20\% receives more than 60\% (Statistics South Africa 2014). In rural areas, this inequality

522 manifests in a $24.5 \%$ rate of youth stunting, much higher than the nation as a whole. Thus, in a

523 nation like South Africa, there may be an opportunity for mariculture to contribute to a

524 nutritional need, even if the nation has a (relatively) low nutritional opportunity score.

525

526 CONCLUSION

527

528 By identifying important regional patterns in mariculture opportunity across three key combined 529 measures of nutritional opportunity, economic opportunity, and seafood reliance, our analysis

530 frames and focuses the necessary discussion on mariculture development and nutrition. An

531 important finding is that nutritional and economic opportunities overlap in many nations, but

532 come with significant challenges. As mariculture industries develop around the world,

533 management choices will need to be made that balance high-value versus widely affordable

534 species and promote nutrition-focused production expansion through appropriate public policy.

535 Our analysis highlights the places where these policies could be impactful in promoting dual 536 economic and nutritional goals, but further studies are needed on how to effectively capture the

537 opportunities we have identified. What is the appropriate balance for a mariculture development 538 program between production for local consumption versus high-value intensive production for 539 export? How should mariculture species be prioritized for production, given country-specific 540 conditions and nutritional needs? What are the environmental and ecological tradeoffs inherent 541 in mariculture development, and how might development be guided to avoid extensive 542 environmental degradation, further imperiling at-risk coastal populations? These questions are 
543 outside of the scope of this study, but remain essential topics for future research, as they will

544 likely become key guiding principles to further mariculture development worldwide.

545

546 Mariculture production continues to expand and develop globally, and is a promising avenue to

547 meet growing global nutritional challenges. Looking forward, if a development goal is to jointly

548 develop mariculture and improve nutrition, countries with a higher reliance on seafood should be

549 prioritized. In our analysis, countries with a high overall mariculture opportunity score not only

550 have the economic development opportunity and a demonstrated nutritional need, but also the

551 dietary preferences to link the two opportunities. Countries with high relative scores across the

552 three components present potential win-win scenarios - where investing in nutrition and

553 mariculture could have synergistic positive effects. Yet significant policy and institutional

554 barriers remain in bridging the current gap between mariculture development and nutritional

555 improvement. Addressing these barriers to achieve the development goal of improved global

556 nutrition requires careful consideration, else we risk wasting a potentially powerful synergy

557 between mariculture and nutrition opportunity.

558

559

\section{ACKNOWLEDGMENTS}

560

561 The authors would like to thank Bill Kuni and his partner Mary for their generous support of and

562 comments on our work. Additionally, the early stages of this project benefitted immensely from

563 the contributions of Patricia Faúndez-Báez and the helpful comments of many other researchers

564 from the Bren School and the National Center for Ecological Analysis and Synthesis. Finally, the

565 authors would like to thanks two anonymous reviewers for constructive feedback.

566

567

\section{LITERATURE CITED}

568

569

570

571 Asche, F., Bellemare, M.F., Roheim, C., Smith, M.D. and Tveteras, S. (2015a) Fair Enough?

$572 \quad$ Food Security and the International Trade of Seafood. World Development 67, 151-160. 
573 Asche, F., Roheim, C. A. and Smith, M.D. (2015b) Trade intervention: Not a silver bullet to 574 address environmental externalities in global aquaculture. Marine Policy 69, 1-8.

575 Asche, F. and Smith, M.D. (2018). Induced innovation in fisheries and aquaculture. Food Policy $576 \quad$ (In press).

577 Ashe, J.W. (2005) Tourism investment as a tool for development and poverty reduction: The 578 experience in Small Island Developing States (SIDS). 18-20.

579

Bailey, R.L., West, K.P. and Black, R.E. (2015) The epidemiology of global micronutrient 580 deficiencies. Annals of Nutrition and Metabolism 66, 22-33.

581

582

583

584

585

586

587

588

589

590

591

592

593

594

595

596

597

598

Béné, C., Arthur, R., Norbury, H., Allison, E.H., Beveridge, M., Bush, S., Campling, L., Leschen, W., Little, D., Squires, D., Thilsted, S.H., Troell, M., and Williams, M. (2016) Contribution of Fisheries and Aquaculture to Food Security and Poverty Reduction: Assessing the Current Evidence. World Development 79, 177-196.

Béné, C., Barange, M., Subasinghe, R., Pinstrup-Andersen, P., Merino, G., Hemre, G.I. and Williams, M. (2015) Feeding 9 billion by 2050? Putting fish back on the menu. Food Security 7, 261-274.

Beveridge, M.C.M., Thilsted, S.H., Phillips, M.J., Metian, M., Troell, M. and Hall, S.J. (2013) Meeting the food and nutrition needs of the poor: The role of fish and the opportunities and challenges emerging from the rise of aquaculturea. Journal of Fish Biology 83, 1067-1084.

Blasiak, R., Spijkers, J., Tokunaga, K., Pittman, J., Yagi, N. and Österblom, H. (2017) Climate change and marine fisheries: Least developed countries top global index of vulnerability. Plos One 12, e0179632.

Bostock, J., McAndrew, B., Richards, R., Jauncey, K., Telfer, T., Lorenzen, K., Little, D., Ross, L., Handisyde, N., Gatward, I., and Corner, R. (2010) Aquaculture: global status and trends. Philosophical Transactions of the Royal Society B: Biological Sciences 365, 2897-2912.

Boyd C, Tucker C, McNevin A, Bostick K, Clay J (2007) Indicators of resource use efficiency and environmental performance in fish and crustacean aquaculture. Reviews in Fisheries 
600 Cao, L., Wang, W., Yang, Y., Yang, C., Yuan, Z., Xiong, S., and Diana, J. (2007) Environmental 601 impact of aquaculture and countermeasures to aquaculture pollution in China. Env Sci $602 \quad$ Pollut Res 14, 452-462.

603 Cole, D.W., Cole, R., Gaydos, S.J., Gray, J, Hyland, G., Jacques, M. L., Powell-Dunford, N., 604 Sawhney, C., and Au, William W. (2009) Aquaculture: Environmental, toxicological, and 605 health issues. International journal of hygiene and environmental health 212, 369-377.

606 Edwards, P. (2015) Aquaculture environment interactions: Past, present and likely future trends. 607 Aquaculture 447, 2-14.

608 FAO (2016) The State of World Fisheries and Aquaculture 2016. Rome.

609 Fiedler, J.L., Lividini, K., Drummond, E. and Thilsted, S.H. (2016) Strengthening the 610 contribution of aquaculture to food and nutrition security: The potential of a vitamin A-rich, 611 small fish in Bangladesh. Aquaculture 452, 291-303.

612 Gentry, R.R., Froehlich, H.E., Grimm, D., Kareiva, P, Parke, M., Rust, M., Gaines, S. D., and 613 Halpern, B. S. (2017) Mapping the global potential for marine aquaculture. Nature Ecology 614 \& Evolution 1.

615 Glencross, B. (2009) Exploring the nutritional demand for essential fatty acids by aquaculture $616 \quad$ species. Reviews in Aquaculture 1, 71-124

617 Golden, C.D., Allison, E.H., Dey, M.M., Halpern, B.S., McCauley, D.J., Smith, M., Vaitla, B., 618 Zeller, D., and Myers, S.S. (2016) Fall in fish catch threatens human health. Nature News $619 \quad \mathbf{5 3 4}, 317-320$.

620 Golden, C.D., Seto, K.L., Dey, M.M., Chen, O.L., Gephart, J., Myers, S.S., Smith, M., Vaitla, B., 621 and Allison, E.H. (2017) Does Aquaculture Support the Needs of Nutritionally Vulnerable 622 Nations? Frontiers in Marine Science 4, 159.

623 Hixon, S. (2014) Fish Nutrition and current issues in aquaculture: The balance in providing safe 
624

625

626

627

628

629

630

631

632

633

634

635

636

637

638

639

640

641

642

643

644

645

646

647

648

and nutritious seafood, in an environmentally sustainable manner. Journal of Aquaculture Research \& Development 5, 234.

Holmer, M. (2010) Environmental issues of fish farming in offshore waters: Perspectives, concerns and research needs. Aquaculture Environment Interactions 1, 57-70.

Heuer, O.E., Kruse, H., Grave, K., Collignon, P., Karunasagar, I., Angulo, F.J. (2008) Human health consequences of use of antimicrobial agents in aquaculture. Clinical Infectious Diseases 49: 1248-1253.

IFPRI (2016) Global Nutrition Report 2016: From Promise to Impact: Ending malnutrition by 2030. International Food Policy Research Institute, Washington, D.C.

Kapetsky, J.M., Aguilar-Manjarrez, J. and Jenness, J. (2013) A global assessment of offshore mariculture potential from a spatial perspective. Rome.

Kawarazuka, N. and Béné, C. (2011) The potential role of small fish species in improving micronutrient deficiencies in developing countries: building evidence. Public Health Nutrition 14, 1927-1938.

Klinger, D. and Naylor, R. (2012) Searching for Solutions in Aquaculture: Charting a Sustainable Course. Annual Review of Environment and Resources 37, 247-276.

Kobayashi, M., Msangi, S., Batka, M., Vannuccini, S., Dey, M. M., \& Anderson, J. L. (2015) Fish to 2030: The Role and Opportunity for Aquaculture. Aquaculture Economics and Management 19, 282-300.

Ganesh Kumar \& Carole R. Engle (2016) Technological Advances that Led to Growth of Shrimp, Salmon, and Tilapia Farming, Reviews in Fisheries Science \& Aquaculture, 24:2.

Larsen, R., Eilertsen, K.E. and Elvevoll, E.O. (2011) Health benefits of marine foods and ingredients. Biotechnology Advances 29, 508-518.

Little, D.C., Newton, R.W. and Beveridge, M.C.M. (2016) Aquaculture: a rapidly growing and significant source of sustainable food? Status, transitions and potential. Proceedings of the 
649

650

651

652

653

654

655

656

657

658

659

660

661

662

663

664

665

666

667

668

669

670

671

672

Nutrition Society 75, 274-286.

May, J. (2016) Why child malnutrition is still a problem in South Africa 22 years into democracy. The Conversation.

Micronutrient Initiative (2009) Investing in the Future: A united call to action on vitamin and mineral deficiencies. Ottawa, Ontario.

National Research Council (2011) Nutritional requirements of fish and shrimp. National Academies Press, Washington.

Ottinger, M., Clauss, K. and Kuenzer, C. (2016) Aquaculture: Relevance, distribution, impacts and spatial assessments - A review. Ocean and Coastal Management 119, 244-266.

Scheyvens, R. and Momsen, J.H. (2008) Tourism and poverty reduction: Issues for small island states. Tourism Geographies 10, 22-41.

Smith, M.R., Micha, R., Golden, C.D., Mozaffarian, D. and Myers, S.S. (2016) Global Expanded Nutrient Supply (GENuS) Model: A New Method for Estimating the Global Dietary Supply of Nutrients. PloS one 11, e0146976.

Statistics South Africa (2014) Poverty Trends in South Africa: An examination of absolute poverty between 2006 and 2011.

Thilsted, S.H., Thorne-Lyman, A., Webb, P., Bogard, J.R., Subasinghe, R., Phillips, M.J. and Allison, E.H. (2016) Sustaining healthy diets: The role of capture fisheries and aquaculture for improving nutrition in the post-2015 era. Food Policy 61, 126-131.

Troell, M., Naylor, R.L., Metian, M., Beveridge, M., Tyedmers, P.H., Folke, C., Arrow, K.J., Barrett, S., Crépin, A., Ehrlich, P.R., Gren, A., Kautsky, N., Levin, S.A., Nyborg, K., Österblom, H., Polasky, S., Scheffer, M., Walker, B.H., Xepapadeas, T., and de Zeeuw, A.. (2014) Does aquaculture add resilience to the global food system? Proceedings of the National Academy of Sciences 111, 13257-13263. 
673 Tveteras, S., Asche, F., Bellemare, M.F., Smith, M.D., Guttormsen, A.G., Lem, A., Lien, K., and 674 Vannuccini, S. (2012) Fish Is Food - The FAO’s Fish Price Index. PloS one 7.

675 UNDP (2015) Human Development Report: work for human development. 1-7.

676 Verdegem, M.C.J. and Bosma, R.H. (2009) Water withdrawal for brackish and inland 677 aquaculture, and options to produce more fish in ponds with present water use. Water Policy 67811.

679 Watson, R.A., Green, B.S., Tracey, S.R., Farmery, A. and Pitcher, T.J. (2016) Provenance of 680 global seafood. Fish and Fisheries 17, 585-595.

681 Watson, R., Nowara, G.B., Hartmann, K., Green, B.S., Tracey, S.R. and Carter, C.G. (2015)

682 Marine foods sourced from farther as their use of global ocean primary production 683 increases. Nature Communications 6, 736. 
Figure 1 (on next page)

Schematic of mariculture opportunity score calculation for each nation.

Categories of metrics consolidated for clarity, with total number of raw variables in parentheses. See Equations 2-5 for score calculation. 
Seafood trade balance (2)

Opportunity scores

Current production relative to fisheries

Willingness to pay (1)

GDP per capita (1)

Nutrients in diet (5)

Energy adequacy (1)

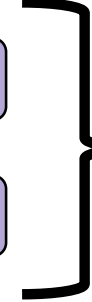

Economic

Opportunity Score
Mariculture Opportunity Score
Percent of nutrients from seafood (6)
Nutritional

Opportunity Score
Seafood

Reliance

Score 


\section{Figure 2 (on next page)}

(A) Final mariculture opportunity scores for the entire world, with (B) detail for the Caribbean region.

Gray indicates countries which were removed from the analysis (see Methods) or no data were available. World borders dataset from thematicmapping.org obtained under a creative commons license. 


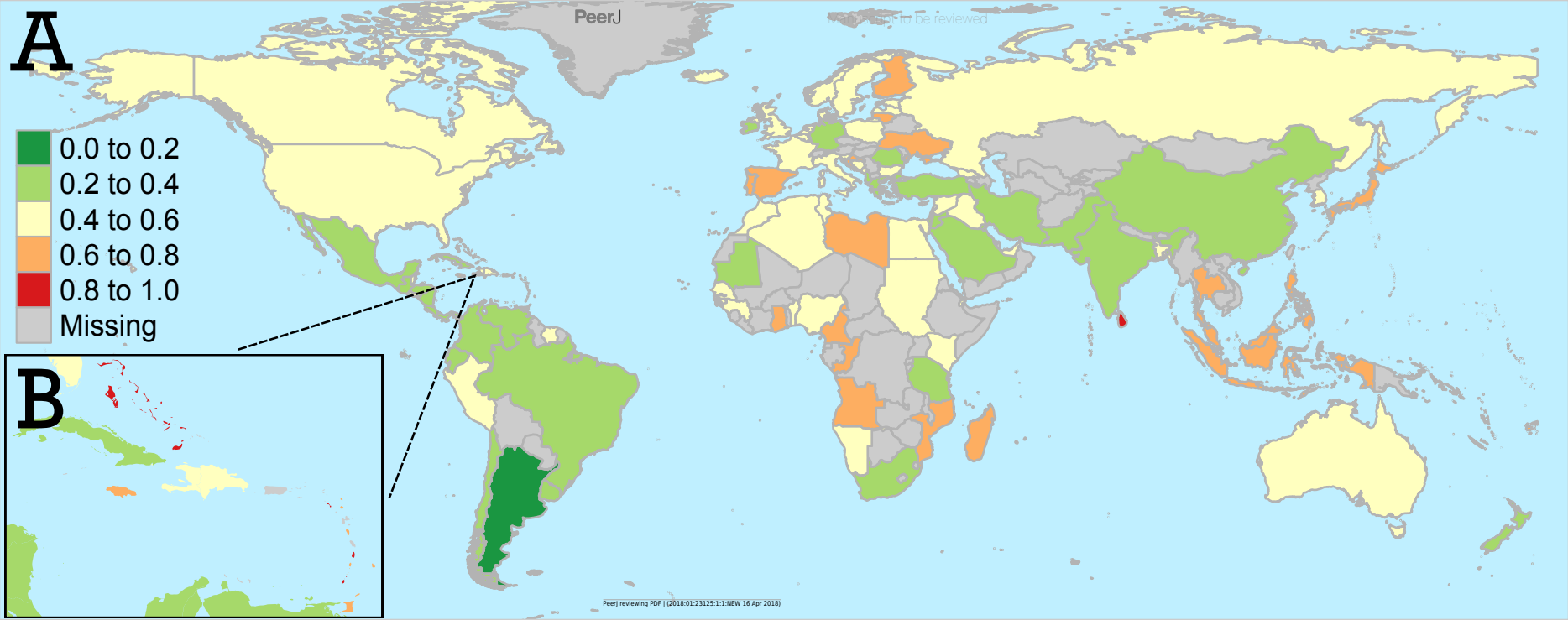




\section{Figure 3 (on next page)}

Results of global analysis of nutritional opportunity, seafood reliance, and economic opportunity.

Individual countries $(n=117)$ scatter along economic and nutritional opportunity scores on the $x$ and $y$ axes, respectively, where each point indicates the performance of a given country. Scores are scaled from zero to one (see Methods), such that countries in the upper right quadrant have both a high economic and nutritional opportunity for mariculture development. Size and opacity of country points scale with each country's seafood reliance score, while color indicates a country's geographic region. Countries referred to in the Discussion are labeled. 


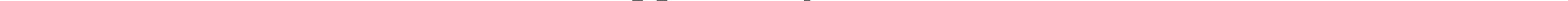

